PROCEEDINGS OF THE

AMERICAN MATHEMATICAL SOCIETY

Volume 126, Number 8, August 1998, Pages 2229-2230

S 0002-9939(98)04580-8

\title{
THE EXISTENCE AND UNIQUENESS OF INJECTORS FOR FITTING SETS OF SOLVABLE GROUPS
}

\author{
IAN HAWTHORN
}

(Communicated by Ronald M. Solomon)

\begin{abstract}
A short alternative proof is given of the existence and uniqueness of injectors in a Fitting set which avoids use of the Carter subgroup, a concept from the dual theory of projectors.
\end{abstract}

A Fitting set $\mathcal{X}$ of a group $G$ is a non-empty set of subgroups which is closed under normal product, subnormal inheritance, and conjugation in $G$. If $H \leq G$, then the groups from $\mathcal{X}$ which are subgroups of $H$ form a Fitting set of $H$ which, since there is seldom danger in confusion, we also call $\mathcal{X}$. An $\mathcal{X}$-injector $S$ of $G$ is a subgroup with the property that $S \cap H$ is $\mathcal{X}$-maximal in $H$ for all $H \unlhd G$. If $G$ is solvable, then $\mathcal{X}$-injectors exist uniquely up to conjugacy.

This theorem was first proved by Fischer, Gaschütz and Hartley in [3]. Their proof can also be found in [2], which also provides a comprehensive introduction to solvable group theory. The core of the proof is a clever lemma of Hartley which uses Carter subgroups (projectors for the class of nilpotent groups).

It is unsatisfying to have the proof of this fundamental result dependent on the corresponding result from the dual theory of projectors. We would prefer a proof based on more elementary ideas. Unfortunately alternative proofs [1] are often long, and our preference is not strong grounds for discarding a short proof in favor of a longer one. Here, however, is a short and simple proof of this type.

Recall that a $p$-subgroup $P_{0} \leq P \in \operatorname{Syl}_{p}(G)$ is strongly closed in $P$ if $P_{0}^{g} \cap P \leq P_{0}$ for all $g \in G$. The proof uses two simple lemmas. The first lemma states that strongly closed $p$-subgroups of solvable groups are normally embedded. This is well known (for example, see [4]). The second lemma is a special case of Corollary $\mathrm{I}(7.11)$ in [2], originally due to Schaller. Neither lemma is particularly difficult to prove.

Lemma 1. Let $G$ be a solvable group. Let $P_{0}$ be strongly closed in $P \in \operatorname{Syl}_{p}(G)$. Then there exists $N \unlhd G$ with $N \cap P=P_{0}$.

Proof. Induct on $|G|$. Let $1 \neq M \unlhd G$ and let $\bar{H}$ denote $H M / M$ for $H \leq G$. Since $\overline{P_{0}}$ is strongly closed in $\bar{P}$ with respect to $\bar{G}$, by hypothesis $\overline{P_{0}}=\bar{P} \cap \bar{N}$ for $\bar{N} \unlhd \bar{G}$. Hence there exists $N \unlhd G$ with $N \cap P=P_{0}(M \cap P)$. If $O_{p^{\prime}}(G) \neq 1$ we can set $M=O_{p^{\prime}}(G)$, and we are done. If $O_{p}(G) \cap P_{0} \neq 1$ we can set $M=O_{p}(G) \cap P_{0}$, and we are done. But now $P_{0} \leq C_{G}\left(O_{p}(G)\right) \leq O_{p}(G)$ by Phillip Hall's theorem (6.1.3 in [5]), and so $P_{0}=P_{0} \cap O_{p}(G)=1$.

Received by the editors January 24, 1997.

1991 Mathematics Subject Classification. Primary 20D10.

(C)1998 American Mathematical Society 
Lemma 2. Let $G$ be a solvable group. Let $P_{0}$ be strongly closed in $P \in \operatorname{Syl}_{p}(G)$ and $Q_{0}$ be strongly closed in $Q \in \operatorname{Syl}_{q}(G)$. Then there exist conjugates of $P_{0}$ and $Q_{0}$ whose product is a group. Equivalently there exist $s, t \in G$ with $P_{0}^{s} Q_{0}^{t}=Q_{0}^{t} P_{0}^{s}$.

Proof. Choose conjugates to ensure that $P$ and $Q$ are from a Sylow system, and hence $P Q=Q P$ is a group. Choose (last lemma) normal subgroups $N$ and $M$ with $N \cap P=P_{0}$ and $M \cap Q=Q_{0}$. But now $P_{0} Q_{0}=P M \cap P Q \cap N Q$, and this is a subgroup as claimed.

Theorem 3 (Fischer, Gaschütz, Hartley). If $G$ is a solvable group and $\mathcal{X}$ is a Fitting set of $G$, then $G$ has a unique conjugacy class of $\mathcal{X}$-injectors.

Proof. Induct on $|G|$. Assume the result for all groups of smaller order. Choose $O^{p}(G)<G$. Let $S$ be an $\mathcal{X}$-injector of $O^{p}(G)$, and let $P \in \operatorname{Syl}_{p}\left(N_{G}(S)\right)$. By the Frattini argument, $G=N_{G}(S) \cdot O^{p}(G)$, hence $P O^{p}(G)=G$.

If $S \leq T \in \mathcal{X}$ then $S \leq T \cap O^{p}(G) \in \mathcal{X}$; hence $T \cap O^{p}(G)=S$ and $T \leq N_{G}(S)$. A Sylow $p$-subgroup of $T$ is conjugate in $N_{G}(S)$ to a subgroup of $P$. Hence $T$ is conjugate in $N_{G}(S)$ to a group of the form $P_{0} S \in \mathcal{X}$, where $P_{0} \leq P$. All such groups are subnormal in $P S$, so the group $R$ that they generate is in $\mathcal{X}$, and is the unique maximal $\mathcal{X}$-subgroup of $P S$. Furthermore, all extensions of $S$ which are elements of $\mathcal{X}$ are conjugate in $N_{G}(S)$ to subgroups of $R$. In particular, if $G$ has an $\mathcal{X}$-injector, then it is conjugate to $R$.

It remains only to show that $R$ is an $\mathcal{X}$-injector. Since $R$ is $\mathcal{X}$-maximal in $G$, it is enough to prove that $R$ contains (and hence $R \cap M$ is equal to) an $\mathcal{X}$-injector of $M$ for every maximal normal subgroup $M$ of $G$.

Suppose $[G: M]=q$, and let $T$ be an $\mathcal{X}$-injector of $M$. Since $T \cap O^{p}(G)$ and $S \cap M$ are $\mathcal{X}$-injectors of $M \cap O^{p}(G)$, we may choose $T$, so that $T \cap O^{p}(G)=$ $S \cap M=U$. Let $P_{1} \in \operatorname{Syl}_{p}(T)$ and $Q_{1} \in \operatorname{Syl}_{q}(S)$, so that $T=P_{1} U$ and $S=Q_{1} U$. Choose $P_{1} \leq P \in \operatorname{Syl}_{p}\left(N_{G}(U)\right)$ and $Q_{1} \leq Q \in \operatorname{Syl}_{q}\left(N_{G}(U)\right)$. If $g \in N_{G}(U)$, then $\left(P_{1}^{g} \cap P\right) U \leq T^{g} \in \mathcal{X}$ and $\left\langle P_{1}^{g} \cap P, P_{1}\right\rangle U \in \mathcal{X}$. Then $P_{1}^{g} \cap P \leq P_{1}$ and $P_{1}$ is strongly closed in $P$ with respect to $N_{G}(U)$. Similarly we can show that $Q_{1}$ is strongly closed in $Q$ with respect to $N_{G}(U)$. So by the last lemma $P_{1}^{g} Q_{1}$ is a group for some $g \in N_{G}(U)$.

Let $K=P_{1}^{g} Q_{1} U=\left(P_{1} U\right)^{g}\left(Q_{1} U\right)=T^{g} S$. Since $K \cap O^{p}(G)=S$ and $K \cap M=T^{g}$, we see that $K=T^{g} S$ is a normal product, and thus $S \leq K \in \mathcal{X}$. Hence $R$ contains a conjugate of $K \geq T$, as claimed.

\section{REFERENCES}

1. T.R. Berger, unpublished draft manuscript.

2. Klaus Doerk and Trevor Hawkes, Finite Soluble Groups, De Gruyter expositions in mathematics 4, 1992. MR 93k:20033

3. B. Fischer, W. Gaschütz and B. Hartley, Injektoren endlicher auflösbarer Gruppen, Math. Z. 102 (1967), 337-339. MR 36:6504

4. David M. Goldschmidt, Strongly closed 2-subgroups of finite groups, Ann. of Math. (2) 102 (1975), no. 3, 475-489. MR 52:14033

5. Daniel Gorenstein, Finite Groups, Second edition, Chelsea, New York, 1980. MR 81b:20002

Department of Mathematics, University of Waikato, New Zealand

E-mail address: hawthorn@waikato.ac.nz 